\title{
Risk factors for retinal breaks during macular hole
} surgery

This article was published in the following Dove Press journal:

Clinical Ophthalmology

\author{
Masashi Sakamoto \\ Izumi Yoshida \\ Ryuya Hashimoto \\ Hidetaka Masahara \\ Takatoshi Maeno \\ Department of Ophthalmology, Toho \\ University Sakura Medical Center, \\ Sakura, Japan
}

Purpose: To identify risk factors for retinal breaks during macular hole ( $\mathrm{MH})$ surgery.

Patients and methods: This retrospective, observational, comparative study included the medical records of 364 consecutive patients (382 eyes) who underwent vitrectomy for $\mathrm{MH}$ at Toho University Sakura Medical Center between January 2012 and May 2017. The patients comprised 135 men and 229 women with mean age 67.6 \pm 8.0 years. Six eyes with a pre-existing retinal tear treated by laser photocoagulation before $\mathrm{MH}$ surgery were excluded. Data on sex, age, presence or absence of lattice degeneration, axial length (measured using an IOL Master ${ }^{\circledR}$ ), preoperative refraction, whether or not combined lens extraction/vitrectomy had been performed, whether or not the lens was pseudophakic before vitrectomy, and whether or not a posterior vitreous detachment had been created intraoperatively were collected. The lattice degeneration status was classified as none (0), treated using laser photocoagulation (1), or untreated (2).

Results: The only item identified in logistic regression analysis with the backward elimination method to be a significant risk factor for retinal breaks during MH surgery was the presence of lattice degeneration $(P<0.001)$.

Conclusion: Ophthalmologists should be aware of the increased risk of retinal breaks during MH surgery in eyes with lattice degeneration. Intraoperative retinal breaks may be less likely to occur in eyes with lattice degeneration treated by photocoagulation preoperatively.

Keywords: macular hole, iatrogenic retinal breaks, vitrectomy, lattice degeneration

\section{Introduction}

Idiopathic macular hole $(\mathrm{MH})$ is common in the elderly, particularly women, and leads to loss of central vision, distortion of vision, and scotoma. ${ }^{1-3} \mathrm{MH}$ is conventionally staged using the classification devised by Gass. ${ }^{4}$ Recent optic coherence tomography studies have suggested that the etiology of $\mathrm{MH}$ involves vitreofoveal traction. ${ }^{4-9}$ If left untreated, the diameter of the $\mathrm{MH}$ increases and visual acuity worsens.

In recent years, vitrectomy with internal limiting membrane (ILM) staining using triamcinolone acetonide, ${ }^{10}$ indocyanine green, ${ }^{11}$ and Brilliant Blue $\mathrm{G}^{12}$ and the inverted flap technique ${ }^{13}$ has been demonstrated to be an effective intervention for $\mathrm{MH}$ but may be complicated by postoperative retinal detachment (RD). ${ }^{14-16}$ Postoperative RD is attributed to breaks in the periphery of the retina caused by creation of posterior vitreous detachment (PVD) and manipulation of instruments at the sclerotomy site during surgery. ${ }^{17-22}$

The incidence of iatrogenic retinal breaks in patients with $\mathrm{MH}$ is reported to be $14.6 \%-35.4 \%$ for 20 -gage surgery, ${ }^{23-26} 3.6 \%-18.3 \%$ for 23 -gage surgery, ${ }^{25,27}$ and $17.3 \%$ for 25 -gage surgery. ${ }^{19}$ Given that RD threatens the postoperative visual acuity in these patients, it is important to be able to identify eyes with an increased risk of an intraoperative retinal break. However, there are few reports on the causes of retinal
Correspondence: Masashi Sakamoto Department of Ophthalmology, Sakura Medical Center, Toho University Sakura Medical Center, 564-I Shimoshizu, Sakura, Chiba Prefecture 285-874I, Japan Tel +8 I 4346288 I I

Fax $+8 \mid 434628820$

Email masashi.sakamoto@

med.toho-u.ac.jp 
breaks during MH surgery. The aim of this retrospective, observational, comparative study was to identify the reasons for retinal breaks during vitrectomy for $\mathrm{MH}$.

\section{Patients and methods}

The medical records of 364 consecutive patients (382 eyes) who underwent vitrectomy for $\mathrm{MH}$ at Toho University Sakura Medical Center between January 2012 and May 2017 were reviewed.

The criterion for inclusion in the study was a full-thickness $\mathrm{MH}$ diagnosed using optical coherence tomography. Eyes with a pre-existing retinal tear and eyes in which the axial length was not measured before vitrectomy were excluded.

The patients consisted of 135 men and 229 women with mean age $67.6 \pm 8.0$ years. Data were collected on patient sex and age, presence of lattice degeneration, axial length (measured using an IOL Master ${ }^{\circledR}$, Carl Zeiss, Oberkochen, Germany), preoperative refraction, whether or not combined lens extraction/vitrectomy had been performed, whether or not the lens was pseudophakic before vitrectomy, and whether or not a PVD had been created intraoperatively. The presence of PVD was confirmed by preoperative optical coherence tomography and staining of the vitreous cortex by triamcinolone acetonide during vitrectomy. The lattice degeneration status was classified as none $(0)$, treated using laser photocoagulation (1), or untreated (2).

The study protocol was reviewed and approved by the ethics committee at Toho University Sakura Medical Center (approval number No S18002). All study conduct adhered to the tenets of the Declaration of Helsinki. Written informed consent was obtained after the study design and risks, and benefits of participation were explained via the Toho University Sakura Medical Center website in accordance with the guidelines for clinical research set out by the Japanese Ministry of Health, Labor, and Welfare prior to initiation of any treatment. All information that could identify patients was removed from the database. Use of the anonymized data was approved by our institutional review board without the need to seek additional consent for use of the data for research purposes.

\section{Statistical analyses}

All statistical analyses were performed using SPSS statistical software (version 24.0, IBM Corporation, Armonk, NY, USA). Six eyes with a pre-existing retinal tear that was treated using laser photocoagulation before $\mathrm{MH}$ surgery were excluded. Axial length was not measured in 7 eyes that underwent lens-sparing vitrectomy or in 29 eyes that were pseudophakic preoperatively; these cases were excluded from the logistic regression analysis, leaving data for 340 eyes available for analysis. Univariate logistic regression analysis was performed to identify potential risk factors for retinal breaks during MH surgery. Variables that were initially significant were analyzed further in a multivariate regression model with the backward elimination method to identify statistically significant risk factors for retinal breaks. A $P$-value $<0.05$ was considered statistically significant.

\section{Surgical procedure}

Standard pars plana vitrectomy was performed under retrobullbar anesthesia ( $2 \%$ lidocaine and $0.75 \%$ ropivacaine) using a 20-gage system in 2 cases, a 23-gage system in 89 cases, a 25-gage system in 274 cases, and a 27-gage system in 17 cases $\left(\right.$ Accuras $^{\circledR}$ or Constellation ${ }^{\circledR}$, Alcon Laboratories, Inc., Fort Worth, TX, USA). Sclerotomies were placed $3.5 \mathrm{~mm}$ from the limbus if the lens was pseudophakic and $4 \mathrm{~mm}$ from the limbus if the lens was phakic. Core vitrectomy was performed and, if not already present, a PVD was induced by staining the vitreous cortex with triamcinolone acetonide. The aspiration pressure was set between 550 and $600 \mathrm{mmHg}$ during creation of the PVD. The PVD was started from the optic disc and developed to the peripheral retina using the vitrectomy probe in cutter-off mode. Indocyanine green or Brilliant Blue G was used to stain the ILM and facilitate its removal. If the MH was large, the inverted ILM flap technique was also used.

A PVD that extended as far as possible to the vitreous base was created, followed by vitreous shaving with scleral indentation. Any untreated retinal holes or tears and lattice degeneration were treated by endolaser photocoagulation followed by gas tamponade using $20 \%$ sulfur hexafluoride.

\section{Results}

According to the classification devised by Gass, the MH was stage $1 \mathrm{~b}$ in 13 eyes, stage 2 in 79 eyes, stage 3 in 192 eyes, and stage 4 in 98 eyes. Ninety-eight $(25.7 \%)$ of the 382 eyes had pre-existing PVD. Closure of the MH postoperatively was confirmed in 361 (94.5\%) of the 382 eyes; iatrogenic intraoperative retinal breaks were detected in 80 eyes $(20.9 \%$; Table 1). We identified two types of breaks, that is, entry-site breaks (ESB) and breaks elsewhere (meaning breaks that were located away from any of sclerotomies). ESB were defined as any new breaks occurring within 1 clock hour of an entry site. Thirteen eyes (16.3\%) had ESB, 53 (66.2\%) had breaks elsewhere, and 14 (17.5\%) had both ESB and breaks elsewhere. Thirty-nine eyes $(48.7 \%)$ with breaks 
Table I Patient demographics and ophthalmic characteristics

\begin{tabular}{|l|l|l|}
\hline Male & $135 / 364$ & $37.1 \%$ \\
\hline Age (years) & $67.6 \pm 8.0$ & \\
\hline Right eye & $196 / 382$ & $51.3 \%$ \\
\hline Pre-existing PVD & $98 / 382$ & $25.7 \%$ \\
\hline Lens resection during vitrectomy & $346 / 382$ & $90.6 \%$ \\
\hline Lens sparing during vitrectomy & $7 / 382$ & $1.8 \%$ \\
\hline Pseudophakic before vitrectomy & $29 / 382$ & $7.6 \%$ \\
\hline Lattice & $21 / 382$ & $5.5 \%$ \\
\hline Lattice treated by photocoagulation & $3 / 382$ & $0.8 \%$ \\
\hline Pre-existing tear & $6 / 382$ & $1.6 \%$ \\
\hline Intraoperative retinal breaks & $80 / 382$ & $20.9 \%$ \\
\hline Axial length (340 cases) (mm) & $24.00 \pm 1.68$ & \\
\hline Preoperative refraction (D) & $-1.21 \pm 3.66$ & \\
\hline
\end{tabular}

Note: Data are presented as mean \pm SD unless otherwise specified.

Abbreviation: PVD, posterior vitreous detachment.

had a single tear and $41(51.3 \%)$ had multiple tears. Retinal breaks occurred in 2 eyes in 2 cases (100\%) using a 20-gage system, 23 eyes in 89 cases (25.8\%) using a 23 -gage system, 54 eyes in 274 cases (19.7\%) using a 25 -gage system, and 1 eye in 17 cases (5.9\%) using a 27 -gage system. When the locations of the retinal breaks were classified into quadrants, 11 eyes (13.7\%) had retinal breaks in the superior quadrant, $21(26.3 \%)$ in the inferior quadrant, $13(16.2 \%)$ in the nasal quadrant, and $12(15.0 \%)$ in the temporal quadrant. Twentythree eyes $(28.8 \%)$ had multiple tears located in more than two quadrants.

Twenty-one eyes had lattice degeneration and $17(81.0 \%)$ of these 21 eyes had retinal breaks during surgery. These breaks occurred at the edge of the lattice degeneration in all cases. Univariate logistic regression analysis identified that lattice degeneration was a risk factor for intraoperative retinal breaks and that retinal breaks occurred significantly more often in eyes with untreated lattice degeneration that in those with lattice degeneration treated by laser photocoagulation $(P<0.001)$. The backward elimination method yielded the same result $(P<0.001 ;$ Table 2$)$.

\section{Discussion}

Intraoperative retinal breaks during $\mathrm{MH}$ surgery are thought to be caused by intraoperative creation of a PVD, lattice degeneration, and manipulation of instruments at the sclerotomy site. ${ }^{17-22,24-27}$ However, it has been suggested that rhegmatogenous $\mathrm{RD}$ is caused by advancing age, myopia, a longer axial length, pseudophakia, and lattice degeneration. ${ }^{28-30}$ There has been a report suggesting that intraoperative endolaser photocoagulation for lattice degeneration reduces the likelihood of RD after MH surgery. ${ }^{17}$ Furthermore, prophylactic photocoagulation can reduce the risk of lattice degeneration in the fellow eye in patients with $\mathrm{RD},{ }^{31-35}$ but might not reduce the risk of RD in the fellow eye. ${ }^{36}$ Given these observations, we sought to identify potential risk factors for retinal breaks during MH surgery. The variables considered were patient sex and age, lattice degeneration, axial length, preoperative refraction, combined lens extraction/ vitrectomy, pseudophakia before MH surgery, and intraoperative creation of a PVD.

We identified lattice degeneration to be a significant risk factor for retinal breaks during MH surgery and that preoperative laser photocoagulation for lattice degeneration decreased this risk. Some reports have suggested a relationship between lattice degeneration and rhegmatogenous $\mathrm{RD} ; ;^{31-35}$ however, prophylactic photocoagulation is unlikely to reduce the risk of spontaneous RD. ${ }^{36}$

Table 2 Results of logistic regression analysis with the backward elimination method to identify risk factors for retinal breaks during macular hole surgery

\begin{tabular}{|c|c|c|c|c|c|c|}
\hline & \multicolumn{4}{|c|}{ Logistic regression analysis } & \multicolumn{2}{|c|}{$\begin{array}{l}\text { Backward elimination } \\
\text { method }\end{array}$} \\
\hline & Variable & $\begin{array}{l}\text { Partial regression } \\
\text { coefficient }\end{array}$ & OR $(95 \% \mathrm{Cl})$ & $P$-value & $\begin{array}{l}\text { Partial regression } \\
\text { coefficient }\end{array}$ & $P$-value \\
\hline Age (years) & & -0.0194 & $0.98(0.95-1.01)$ & 0.2179 & & \\
\hline Sex & Male: 0, Female: I & -0.3847 & $0.68(0.4 I-I . I 2)$ & 0.1308 & & \\
\hline Presence of PVD & Absence: 0, Presence: I & -0.5770 & $0.56(0.30-1.01)$ & 0.0729 & -0.6050 & 0.1083 \\
\hline Presence of lattice degeneration & $\begin{array}{l}\text { None: } 0 \text {, Treated: I, } \\
\text { Untreated: } 2\end{array}$ & 1.7150 & $5.56(2.83-10.92)$ & $<0.001$ & 1.6610 & $<0.001$ \\
\hline Lens sparing during vitrectomy & Absence: 0, Presence: I & $0.384 I$ & $\mathrm{I} .47(0.28-7.7 \mathrm{I})$ & 0.6499 & & \\
\hline Pseudophakic before vitrectomy & Absence: 0, Presence: I & -0.2972 & $0.74(0.27-2.01)$ & 0.5588 & & \\
\hline Axial length (mm) & & -0.0022 & $1.00(0.86-1.16)$ & 0.9769 & & \\
\hline Preoperative refraction (D) & & 0.0459 & $1.05(0.97-1.13)$ & 0.2493 & & \\
\hline
\end{tabular}

Abbreviation: PVD, posterior vitreous detachment. 
It is possible that the mechanical traction exerted on the area of lattice degeneration by vitrectomy during $\mathrm{MH}$ surgery is stronger than the vitreoretinal traction exerted by spontaneous RD. Furthermore, the vitreoretinal traction caused by manipulation of instruments at the sclerotomy site during MH surgery might pull on the area of lattice degeneration and cause retinal breaks. This pull might not be so strong if the areas of lattice degeneration are treated preoperatively by laser photocoagulation, thereby reducing the risk of retinal breaks.

Pseudophakia affects the likelihood of RD, and there is a report showing that vitreoretinal traction in eyes with a ruptured posterior capsule causes RD if vitreous resection is incomplete during cataract surgery. ${ }^{37}$ However, if the posterior capsule is ruptured during $\mathrm{MH}$ surgery, the vitreous traction is less likely to affect the retina because the traction can be released by intraoperative resection of the vitreous.

In eyes with a longer axial length, it is considered that the case might have the degeneration on the peripheral retina because of the thin retina. A longer axial length increases the risk of $\mathrm{RD}$, but was not found to cause retinal breaks during MH surgery in our study. Further studies are needed to determine if there is a relationship between longer axial length and lattice degeneration.

This retrospective study has several limitations that should been borne in mind when interpreting its findings. First, we could not investigate the risk of intraoperative retinal breaks according to the gage system used. There is some evidence that use of a smaller gage size for sclerotomy is less likely to cause intraoperative retinal breaks during vitrectomy; ${ }^{18}$ in our study, use of a smaller gage size for sclerotomy was less likely to cause retinal breaks during surgery. However, a 20-gage system was only used in two cases in our study so we could not investigate this relationship. The relationship between gage size and the likelihood of a retinal break during MH surgery should be investigated in the future. Second, we cannot exclude the possibility that we did not identify all retinal breaks that occurred during MH surgery. We searched carefully for retinal breaks with scleral indentation intraoperatively, but may have missed small retinal tears. In the current study, there were not RD immediately after MH surgery, we need to observe for longer time to search the postoperative RD. Third, we could not assess the size of the MH. However, we do not believe that the size of the MH was related to the occurrence of intraoperative retinal breaks; we consider that the size of the $\mathrm{MH}$ is more likely to be related to the duration of $\mathrm{MH}$ than to the area of the peripheral retina where the retinal break occurred intraoperatively. The relationship between the size of a $\mathrm{MH}$ and a retinal break that occurs during vitrectomy needs to be investigated further. Fourth, intraoperative retinal breaks occurred in some eyes without lattice degeneration. In these cases, the aspiration pressure, surgical procedure used to create the PVD, and strength of vitreoretinal adhesion may have contributed to the development of these intraoperative retinal breaks. However, these factors could not be assessed quantitatively, so other parameters should be investigated as potential risk factors for intraoperative retinal breaks during MH surgery. Untreated lattice degeneration may be one such risk factor.

\section{Conclusion}

Intraoperative endolaser photocoagulation in areas of lattice degeneration may reduce the likelihood of RD after MH surgery. Preoperative photocoagulation for lattice degeneration after careful inspection of the ocular fundus may be useful when embarking on MH surgery.

\section{Acknowledgments}

We would like to thank Chiaki Nishimura for statistical advice and Editage for editing this manuscript.

\section{Author contributions}

Design and conduct of the study: MS, IY. Data collection: MS, IY, RH, HM. Management, analysis, and interpretation of the data: MS, IY, TM. Preparation, review, or approval of the manuscript: MS, IY, RH, HM, TM. All authors contributed toward data analysis, drafting and critically revising the paper and agree to be accountable for all aspects of the work.

\section{Disclosure}

The authors report no conflicts of interest in this work.

\section{References}

1. La Cour M, Friis J. Macular holes: classification, epidemiology, natural history and treatment. Acta Ophthalmol Scand. 2002;80(6):579-587.

2. Rahmani B, Tielsch JM, Katz J, et al. The cause-specific prevalence of visual impairment in an urban population. The Baltimore Eye Survey. Ophthalmology. 1996;103(11):1721-1726.

3. Kang HK, Chang AA, Beaumont PE. The macular hole: report of an Australian surgical series and meta-analysis of the literature. Clin Exp Ophthalmol. 2000;28(4):298-308.

4. Gass JD. Idiopathic senile macular hole. Its early stages and pathogenesis. Arch Ophthalmol. 1988;106(5):629-639.

5. Smiddy WE, Flynn HW. Pathogenesis of macular holes and therapeutic implications. Am J Ophthalmol. 2004;137(3):525-537.

6. Benson WE, Cruickshanks KC, Fong DS, et al. Surgical management of macular holes: a report by the American Academy of Ophthalmology. Ophthalmology. 2001;108(7):1328-1335. 
7. Johnson RN, Mcdonald HR, Lewis H, et al. Traumatic macular hole: observations, pathogenesis, and results of vitrectomy surgery. Ophthalmology. 2001;108(5):853-857.

8. Park SS, Marcus DM, Duker JS, et al. Posterior segment complications after vitrectomy for macular hole. Ophthalmology. 1995;102(5): 775-781.

9. Wendel RT, Patel AC, Kelly NE, Salzano TC, Wells JW, Novack GD. Vitreous surgery for macular holes. Ophthalmology. 1993;100(11): 1671-1676.

10. Kimura H, Kuroda S, Nagata M. Triamcinolone acetonide-assisted peeling of the internal limiting membrane. Am J Ophthalmol. 2004; 137(1):172-173.

11. da Mata AP, Burk SE, Riemann CD, et al. Indocyanine green-assisted peeling of the retinal internal limiting membrane during vitrectomy surgery for macular hole repair. Ophthalmology. 2001;108(7):1187-1192.

12. Enaida H, Hisatomi T, Hata Y, et al. Brilliant Blue G selectively stains the internal limiting membrane/brilliant Blue G-assisted membrane peeling. Retina. 2006;26(6):631-636.

13. Michalewska Z, Michalewski J, Adelman RA, Nawrocki J. Inverted internal limiting membrane flap technique for large macular holes. Ophthalmology. 2010;117(10):2018-2025.

14. Kelly NE, Wendel RT. Vitreous surgery for idiopathic macular holes Results of a pilot study. Arch Ophthalmol. 1991;109(5):654-659.

15. Banker AS, Freeman WR, Kim JW, Munguia D, Azen SP. Visionthreatening complications of surgery for full-thickness macular holes. Vitrectomy for Macular Hole Study Group. Ophthalmology. 1997; 104(9):1442-1452.

16. Tabandeh H, Chaudhry NA, Smiddy WE. Retinal detachment associated with macular hole surgery: characteristics, mechanism, and outcomes. Retina. 1999;19(4):281-286.

17. Hwang J, Escariao P, Iranmanesh R, Tosi GM, Chang S. Outcomes of macular hole surgery in patients treated intraoperatively for retinal breaks and/or lattice degeneration. Retina. 2007;27(9):1243-1248.

18. Gosse E, Newsom R, Lochhead J. The incidence and distribution of iatrogenic retinal tears in 20-gauge and 23-gauge vitrectomy. Eye. 2012; 26(1):140-143.

19. Tan HS, Mura M, de Smet MD. Iatrogenic retinal breaks in 25-gauge macular surgery. Am J Ophthalmol. 2009;148(3):427-430.

20. Wimpissinger B, Binder S. Entry-site-related retinal detachment after pars plana vitrectomy. Acta Ophthalmol Scand. 2007;85(7):782-785.

21. Scartozzi R, Bessa AS, Gupta OP, Regillo CD. Intraoperative sclerotomy-related retinal breaks for macular surgery, 20- vs 25-gauge vitrectomy systems. Am J Ophthalmol. 2007;143(1):155-156.

22. Moore JK, Kitchens JW, Smiddy WE, Mavrofrides EC, Gregorio G. Retinal breaks observed during pars plana vitrectomy. Am JOphthalmol. 2007;144(1):32-36.
23. Chung SE, Kim KH, Kang SW. Retinal breaks associated with the induction of posterior vitreous detachment. Am J Ophthalmol. 2009; 147(6):1012-1016.

24. Ramkissoon YD, Aslam SA, Shah SP, Wong SC, Sullivan PM. Risk of iatrogenic peripheral retinal breaks in 20-G pars plana vitrectomy. Ophthalmology. 2010;117(9):1825-1830.

25. Nakano T, Uemura A, Sakamoto T. Incidence of iatrogenic peripheral retinal breaks in 23-gauge vitrectomy for macular diseases. Retina. 2011;31(10):1997-2001.

26. Yagi F, Takagi S, Tomita G. Incidence and causes of iatrogenic retinal breaks in idiopathic macular hole and epiretinal membrane. Semin Ophthalmol. 2014;29(2):66-69.

27. Rahman R, Murray CD, Stephenson J. Risk factors for iatrogenic retinal breaks induced by separation of posterior hyaloid face during 23-gauge pars plana vitrectomy. Eye. 2013;27(5):652-656.

28. Mitry D, Charteris DG, Fleck BW, Campbell H, Singh J. The epidemiology of rhegmatogenous retinal detachment: geographical variation and clinical associations. Br J Ophthalmol. 2010;94(6):678-684.

29. Mitry D, Singh J, Yorston D, et al. The fellow eye in retinal detachment: findings from the Scottish Retinal Detachment Study. Br J Ophthalmol. 2012;96(1):110-113.

30. Potic J, Bergin C, Giacuzzo C, Daruich A, Konstantinidis L, Wolfensberger TJ. Primary rhegmatogenous retinal detachment: risk factors for macular involvement. Graefes Arch Clin Exp Ophthalmol. 2018; 256(3):489-494

31. Folk JC, Arrindell EL, Klugman MR. The fellow eye of patients with phakic lattice retinal detachment. Ophthalmology. 1989;96(1):72-79.

32. Girard P, Goichot L, Saragoussi JJ, Merad I, Forest A. Outcome of the unaffected eye in retinal detachment. Study of 1148 patients. $J$ Fr Ophtalmol. 1982;5(11):681-685, Article in French.

33. Kanski JJ, Daniel R. Prophylaxis of retinal detachment. Am J Ophthalmol. 1975;79(2):197-205.

34. Laatikainen L. The fellow eye in patients with unilateral retinal detachment: findings and prophylactic treatment. Acta Ophthalmol. 1985;63(5):546-551.

35. Mcpherson A, O'Malley R, Beltangady SS. Management of the fellow eyes of patients with rhegmatogenous retinal detachment. Ophthalmology. 1981;88(9):922-934.

36. Wilkinson $\mathrm{CP}$. Interventions for asymptomatic retinal breaks and lattice degeneration for preventing retinal detachment. Cochrane Database Syst Rev. 2014;(9):CD003170CD003170.

37. Petousis V, Sallam AA, Haynes RJ, et al. Risk factors for retinal detachment following cataract surgery: the impact of posterior capsular rupture. Br J Ophthalmol. 2016;100(11):1461-1465.
Clinical Ophthalmology

\section{Publish your work in this journal}

Clinical Ophthalmology is an international, peer-reviewed journal covering all subspecialties within ophthalmology. Key topics include: Optometry; Visual science; Pharmacology and drug therapy in eye diseases; Basic Sciences; Primary and Secondary eye care; Patient Safety and Quality of Care Improvements. This journal is indexed on Submit your manuscript here: http://www.dovepress.com/clinical-ophthalmology-journa

\section{Dovepress}

PubMed Central and CAS, and is the official journal of The Society of Clinical Ophthalmology (SCO). The manuscript management system is completely online and includes a very quick and fair peer-review system, which is all easy to use. Visit http://www.dovepress.com/ testimonials.php to read real quotes from published authors. 